\title{
TEC response to the 2008 Wenchuan Earthquake in comparison with other strong earthquakes
}

E. L. AFRAIMOVICH*†, FENG DING E. I. ASTAFYEVA $\uparrow$, SHUANGGEN JINII and V. A. SANKOV|

$\dagger$ Institute of Solar-Terrestrial Physics SB RAS, P.O. Box 291, Irkutsk, 664033, Russia

tInstitute of Geology and Geophysics, Chinese Academy of Sciences,

Beijing, 100029, China

$\S$ Department of Natural History Sciences, Hokkaido University, Sapporo, Japan, 060-0810, Kita-ku, N10 W8

IIShanghai Astronomical Observatory, Chinese Academy of Sciences, Shanghai, 200030, China

|Institute of the Earth's Crust of SB RAS, Lermontov Street, 128, 664033, Irkutsk, Russia

\begin{abstract}
We registered near-field global positioning system (GPS) total electron content (TEC) response to the Wenchuan Earthquake on 12 May 2008. The Wenchuan Earthquake (magnitude 8.0) occurred at 06:28 UT as the result of motion on a northeast striking reverse fault (thrust fault) on the northwestern margin of the Sichuan Basin. The earthquake reflects tectonic stresses resulting from the convergence of crustal material slowly moving from the high Tibetan Plateau, to the west, against a strong crust underlying the Sichuan Basin and southeastern China. We found that intensive $N$-shaped shock-acoustic waves with a plane waveform and with a half-period of about 200 s propagated south-eastwards with a velocity $580 \mathrm{~m} \mathrm{~s}^{-1}$ for a distance of about $1000 \mathrm{~km}$ from the epicentre. The wavefront of $N$-shaped disturbance was parallel with the earthquake rupture direction (from southwest to northeast). The main directional lobe of shock-acoustic wave emitter is directed southeastwards, i.e. transversely to the rupture. We speculate that the above properties of TEC response are determined by the geodynamics of the Wenchuan Earthquake. No noticeable TEC response to that earthquake was found in far-field regions in South Korea and Japan. We compared TEC response to the 2008 Wenchuan earthquake with other strong earthquakes.
\end{abstract}

\section{Introduction}

The method of global positioning system (GPS) monitoring of the ionosphere is widely used for detecting and studying ionospheric response to earthquakes. For the first time (Calais et al. 1995, 1998) after the earthquake in California on 17 January 1994, anomalous total electron content (TEC) signals were observed in the period range 3-10 min. Frequency and propagation phase velocity $\left(300-600 \mathrm{~m} \mathrm{~s}^{-1}\right)$ agreed with the results of numerical simulation for atmospheric gravity waves (AGW) caused by a rapid uplift or subsidence of the Earth's surface during the earthquake. The velocity value was determined as a ratio of Sub-Ionospheric Point (SIP) distance from the epicentre to the time delay of perturbation arrival to the corresponding SIP

*Corresponding author. Email: afra@iszf.irk.ru

$$
\begin{gathered}
\text { International Journal of Remote Sensing } \\
\text { ISSN 0143-1161 print/ISSN 1366-5901 online (C) } 2010 \text { Taylor \& Francis } \\
\text { http:///www.tandf.co.uk/journals } \\
\text { DOI: } 10.1080 / 01431161003727747
\end{gathered}
$$


(Calais et al. 1995, 1998). Afraimovich et al. (2001a) proposed a simple interferometric method for determining the angular characteristics of the wave vector and phase velocity of $N$-shaped shock-acoustic waves (SAW), generated during earthquakes. They used GPS-arrays, consisting of three sites; the distance was about one-half the wavelength of the perturbation (D1 method). This method provides an estimation of SAW parameters without a priori information about the site and time of an earthquake main shock. It was not possible to determine the form of phase front of SAW (plane or the spherical wavefront) since we used the D1 method.

Afraimovich (2000) first suggested the concept of a new technology for the detection of ionospheric disturbances using GPS arrays. According to this concept, the TEC measurements along the GPS line-of-sight (LOS) were considered as elements of non-equidistant phased antenna arrays of the acoustic detectors located at the ionospheric heights. The TEC measurements were subjected to processing using space-time analysis. On the basis of this concept the different methods of space-time processing of TEC series were developed, including the quasi-optimum algorithm, QOA (Afraimovich et al. 2002a, 2006, Kiryushkin and Afraimovich 2007). These algorithms used the 'array processing' technique and realized the coherent summation of the TEC series within the model of the plane or spherical wavefront accounting for space-time parameters of disturbance. In order to determine parameters of ionospheric perturbation the parameters values were varied. For each combination of the estimated parameters the normalized criterion function $C$ for the coherent sum of all TEC series were calculated. The largest maximum value $C_{\max }$ corresponds to the best-fit perturbation parameters.

In this paper, we used our D1 and QOA methods to study the near-field TEC response to the very strong and deleterious Wenchuan Earthquake that occurred on 12 May 2008. We compared TEC response to the 2008 Wenchuan Earthquake with other strong earthquakes.

\section{General information about the earthquake}

The Wenchuan Earthquake of 12 May 2008 (magnitude 8.0) occurred at 06:28 UT as the result of motion on a northeast striking reverse fault (thrust fault) on the northwestern margin of the Sichuan Basin. The earthquake reflects tectonic stresses resulting from the convergence of crustal material slowly moving from the high Tibetan Plateau, to the west, against a strong crust underlying the Sichuan Basin and southeastern China (http://earthquake.usgs.gov).

The rupture started at the epicentre $\left(31.0^{\circ} \mathrm{N}, 103.4^{\circ} \mathrm{E}\right)$ and over the next $50 \mathrm{~s}$ travelled about $285 \mathrm{~km}$ towards the northeast, tearing apart the land along the front of the mountain range (http://www.tectonics.caltech.edu/). The displacements measured in the field were found to be $6.5 \mathrm{~m}$ vertically, accompanied by an average left-lateral component of $2 \mathrm{~m}$ along the $50 \mathrm{~km}$ long southern most segment of the rupture zone and an average right-lateral component of $1 \mathrm{~m}$ along the $150 \mathrm{~km}$ long central-northern segments. The maximum thrust slip amount is estimated to be $\sim 10 \mathrm{~m}$ (Lin et al. 2009).

Figure 1 shows the location of rupture. Model results of the ruptured fault line obtained from the Caltech Tectonics Observatory (http://www.tectonics.caltech.edu/) are shown in figure 1 by narrow rectangle superimposed on the actual fault line. The earthquake epicentre is shown as a red star. It was found that the rupture propagated from southwest to northeast. Similar results were obtained by Ji and Hayes (2008) 


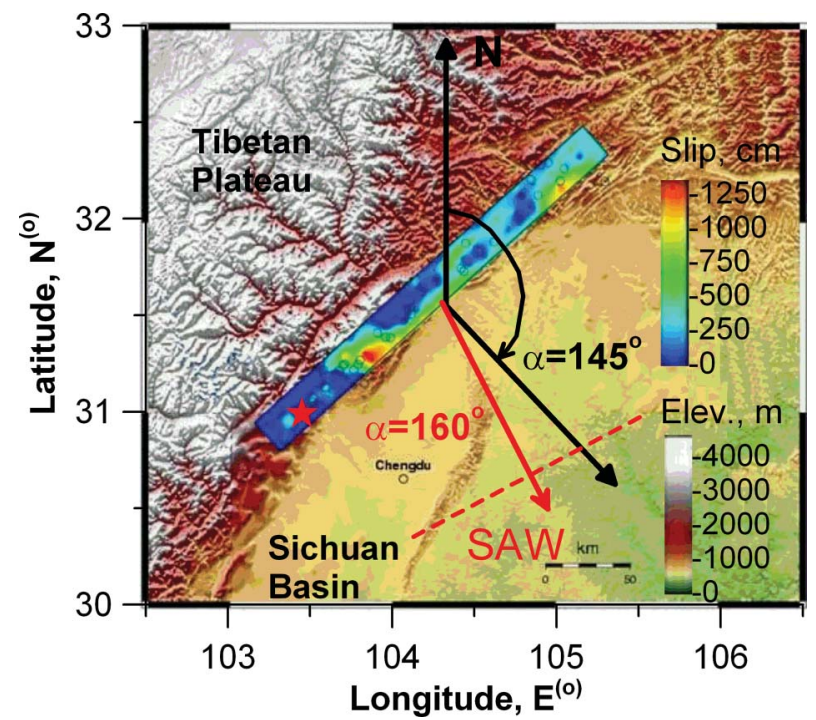

Figure 1. Map of the Wenchuan Earthquake region on 12 May 2008. The red star shows the location of the epicentre. Black and red arrows show the normals to the earthquake rupture (narrow rectangle) and to the plane wavefront (red dashed line) of the excited shock-acoustic wave, respectively. Symbols $\alpha$ and $N$ mark the azimuth of normal and north direction, respectively. Model results of the ruptured fault line obtained from the Caltech Tectonics Observatory (http://www.tectonics.caltech.edu/) are shown by the narrow rectangle superimposed on the actual fault line.

using data of Peak Ground Velocity calculated according to the Finite Fault Model. The last slip model is slightly different, but the rupture direction is also from southwest to northeast.

Recent studies (Zhao et al. 2008, Yu et al. 2009) have been carried out to study the important mechanism of the seismo-ionospheric interactions as a preparation stage for the strong Wenchaun Earthquake. However, the purpose of the present study is to the study the ionospheric response to the main Wenchuan Earthquake and other strong events.

We investigated the response in a near-field of the earthquake epicentre. Unfortunately IGS GPS sites in the near-field of the Wenchuan Earthquake epicentre are relatively sparse. Therefore, we have used GPS data from 27 GPS sites, which belong to the Crustal Movement Observation Network of China. Location of the GPS sites is shown in figure 2 by red triangles (http://www.gfy.ku.dk/ iag/prchina03/ CRUSTAL\%20MOVEMENT\%20OBSERVATION.htm).

We have also made efforts to study the ionospheric response of Wenchuan Earthquake in the far-field regions of the epicentre. We have used data from the Japanese GPS network GEONET (1225 stations) (distribution of the stations is shown in figure 2 in violet). At present, it is the largest regional GPS network in the world (ftp://terras.gsi.go.jp/data/GPS_products/). We have also used data from 50 GPS sites from the South Korean GPS array KGN (Jin and Park 2007) (distribution of these stations is shown in figure 2 in pink). The level of geomagnetic disturbances was quiet during the Wenchuan Earthquake; the $\mathrm{K}_{\mathrm{p}}$ index was found to vary from 0 to 1 on 12 May 2008 (http://www.wdc.rl.ac.uk/cgi-bin/wdccl/secure/wdcdata). 


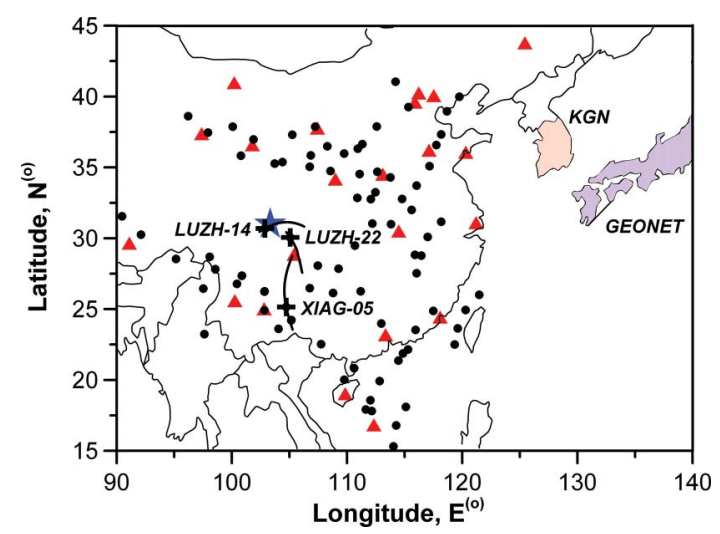

Figure 2. Experimental geometry of global positioning system (GPS) measurements during the Wenchuan Earthquake. The blue star shows the location of the epicentre. The locations of the GPS sites are shown by red triangles. The locations of the sub-ionospheric points (SIP) at time $t_{\max }$ for all used line-of-sites are marked by thick dots. Black crosses represent the D1 GPS array LUZH-PRN14, LUZH-PRN22 and XIAG-PRN05 used. The trucks of the SIPs for selected GPS array are shown by green bold curves. The distributions of the GPS sites of the GEONET and KGN networks in far-field regions are shown in violet and pink, respectively.

\section{GPS data processing and results}

Our methods are described in detail by Afraimovich et al. (2001a) and Kiryushkin and Afraimovich (2007). We now summarize briefly the sequence of data processing procedures and results.

The data used in the present study are available in the standard Receiver Independent Exchange Format (RINEX) with sampling intervals of $30 \mathrm{~s}$. The standard GPS technology provides a means for detecting wave disturbances using phase measurements of the slant TEC $I_{s}$ along the LOS between a GPS site and a satellite. Methods for calculating $I_{s}$ using GPS phase measurements are described in detail by Calais and Minster (1995) and Afraimovich et al. (2001a). We reproduce here only the final equation (1) for phase measurements:

$$
I_{S}(t)=\frac{1}{40.308} \frac{f_{1}^{2} f_{2}^{2}}{\left(f_{1}^{2}-f_{2}^{2}\right)}\left[\left(L_{1} \lambda_{1}-L_{2} \lambda_{2}\right)+\text { const }+n L\right]
$$

where $L_{1} \lambda_{1}$ and $L_{2} \lambda_{2}$ are additional paths of the radio signal caused by the phase delay in the ionosphere (m), $L_{1}$ and $L_{2}$ are numbers of phase rotations at the frequencies $f_{1}$ and $f_{1} ; \lambda_{1}$ and $\lambda_{2}$ stand for the corresponding wavelengths, $(\mathrm{m})$; const is the unknown initial phase ambiguity, (m); and $n L$ is the error in determining the phase path (m).

To normalize the amplitude of TEC disturbances, we use the transformation of slant TEC into the equivalent vertical value $I(t)$ (Klobuchar 1986):

$$
I(t)=I_{s}(t) \cos \left[\arcsin \left(\frac{r_{z}}{r_{z}+h_{\max }} \cos \theta_{s}\right)\right]
$$

where $r_{z}$ is the Earth's radius; $\cos \theta_{s}$ is the LOS elevation to the GPS satellite; $h_{\max }=$ $300-400 \mathrm{~km}$ is the assumed altitude of the ionospheric F2 layer maximum. We neglect the TEC data from low LOS elevation angles (less than $45^{\circ}$ ) to reduce errors 
due to conversion from slant to vertical TEC. Location of the SIPs at time $t_{\max }$ of TEC response to earthquake main shock for all used LOS is marked by thick dots (see below).

To smooth the phase measurement noise and to select TEC variations $d I(t)$ in the range of periods of TEC response to earthquakes (Afraimovich et al. 2001a, 2002b), we first smooth the initial series with a time window of $1 \mathrm{~min}$ and then remove the linear trend with a window of about $10 \mathrm{~min}$. Out of a set of GPS sites, three sites (A, B, C) are selected for D1 method (Afraimovich et al. 2001a). Site B is taken to be the centre of a topocentric reference frame whose axis $x$ is directed east, and axis $y$ is directed north. The receivers in this frame of reference have the coordinates $\left(x_{A}, y_{A}\right),(0,0)$, $\left(x_{c}, y_{c}\right)$.

The trucks of the SIPs for selected GPS sites and GPS satellite numbers LUZHPRN14, LUZH-PRN22 and XIAG-PRN05 are shown in figure 2 as green bold curves. Location of the SIPs at the moments of maximum TEC deviation is shown in figure 2 (black crosses).

The input data include a series of slant TEC values $I_{A}(t), I_{B}(t), I_{C}(t)$, as well as corresponding series of elevation values $\theta_{s}(t)$ and the azimuth $\alpha_{s}(t)$ of the LOS. Elevation $\theta_{s}(t)$ and azimuth $\alpha_{s}(t)$ values of the LOS are used to determine the location of the SIPs.

Figure 3 shows the filtered vertical TEC series $\mathrm{d} I(t)$ for LUZH-PRN14 (a), LUZHPRN22 (b); black, red, and grey lines for 11, 12 and 13 May, respectively. A typical half-period is about 180-210 s. The oscillation amplitude of about 1 TECU (TECU = $\left.10^{16} \mathrm{~m}^{-2}\right)$ is far in excess (10-30 times) of the background TEC fluctuation intensity (Afraimovich et al. 2001b) as seen on the days before (11 May) and after (13 May) the earthquake. In this case, the minimal delay of the SAW response (for LUZH-PRN14) with respect to the time of the earthquake main shock is found to be $10.5 \mathrm{~min}$.

The two TEC series for LUZH-PRN14, LUZH-PRN22 and time dependence $\mathrm{d} I(t)$ for the LOS XIAG-PRN05 are used to determine the velocity and direction of TEC disturbance phase front travelling as three functions $\mathrm{d} I_{\mathrm{A}}(t), \mathrm{d} I_{\mathrm{B}}(t), \mathrm{d} I_{\mathrm{C}}(t)$ (panel $d$ ). Taking into account good signal/noise ratio (much better than 1), and knowing the coordinates of the SIP LUZH-PRN14, LUZH-PRN22 and XIAG-PRN05, we determine the horizontal projection of the phase velocity $V$ from time shifts $t_{\max }$ of a maximum deviation of the filtered TEC series $\mathrm{d} I(t)$. This is followed by a calculation of the $E$ - and $N$-components of $V_{x}$ and $V_{y}$ as well as the direction $\alpha$ in the range of angles $0-360^{\circ}$ and the modulus $V$ of the horizontal component of the SAW phase velocity

$$
\alpha=\arctan \left(V_{y} V_{x}^{-1}\right) ; V=\left|V_{x} V_{y}\right|\left(V_{x}^{2}+V_{y}^{2}\right)^{-1 / 2}
$$

where $V_{x}, V_{y}$ are the velocities of the phase front crossing the axes $x$ and $y$. The azimuth $\alpha$ of the wave vector is counted off from northwards in a clockwise direction.

The azimuth $\alpha$ of the wave vector shown in figure 1 by a thick grey arrow equals $160^{\circ}$. One can see that the phase front of the N-shaped disturbance is parallel with the rupture direction (from southwest to northeast). The phase velocity was found to be $583 \mathrm{~m} \mathrm{~s}^{-1}$.

The QOA algorithm provides the calculation of the normalized criterion function $C$. Function $C$ is the relation of the energy of the GPS-array output signals obtained at a modelling stage to energy obtained at an experimental stage of processing. The maximum value of $C$ is 1.0. At an experimental stage the output signal is formed as 

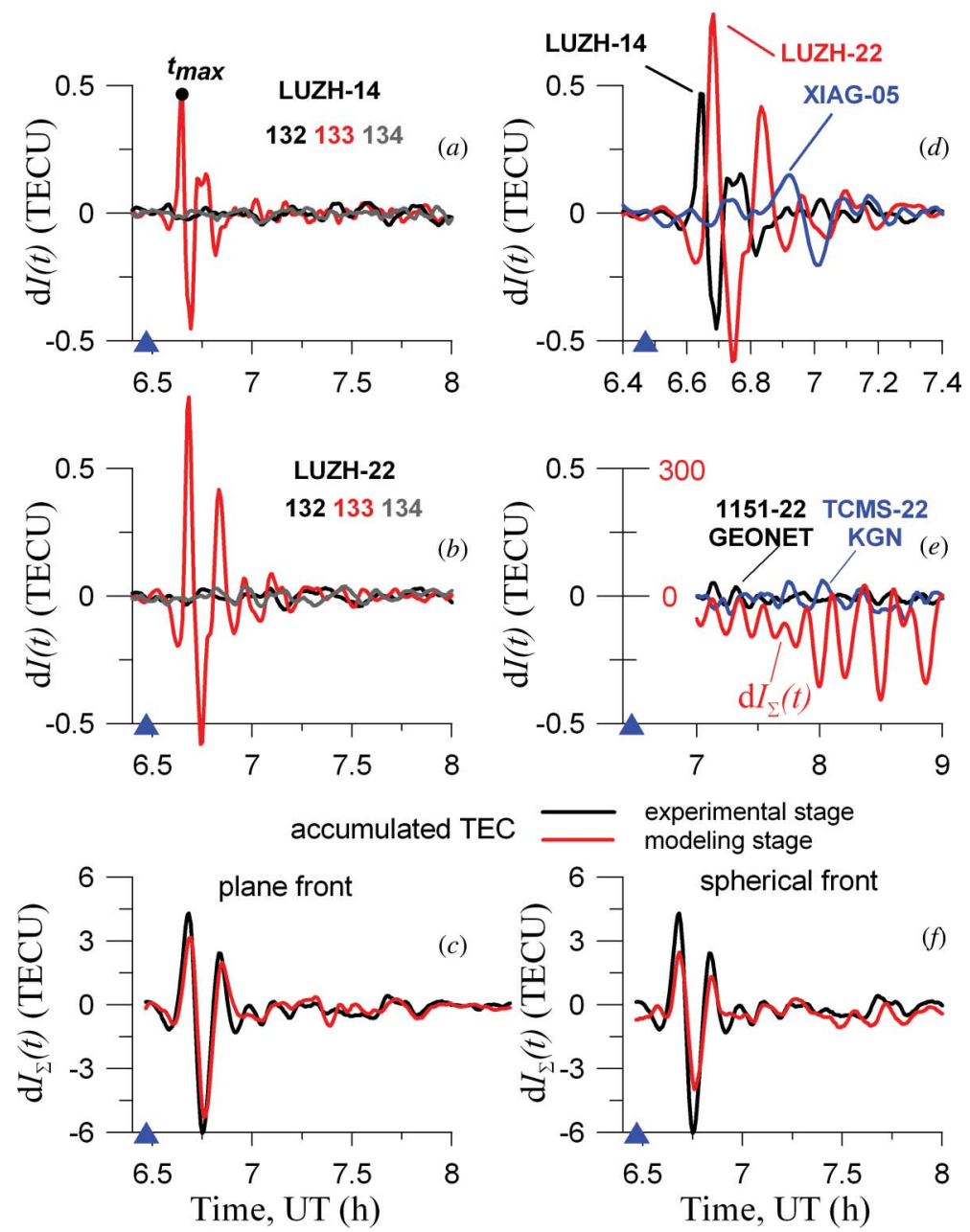

Figure 3. The filtered total electron content (TEC) series d $I(t)$ for LUZH-PRN14 $(a)$, LUZHPRN22 (b); black, red and grey lines - 11, 12 and 13 May, respectively. The filtered TEC series $\mathrm{d} I(t)$ for LUZH-PRN14 (black), LUZH-PRN22 (red) and XIAG-05 (blue) for the D1 GPS array $(d)$. Time moment $t_{\max }$ of a maximum deviation of the $\mathrm{d} I(t)$ is marked by a thick dot $(a)$. $(c),(f)$ The black lines show the accumulated TEC series $\mathrm{d} I_{\Sigma}(t)$, obtained at the experimental stage of processing for the plane and spherical wavefront, respectively (QOA method). The red lines on $(c)$ and $(f)$ mark the modelled $\mathrm{d} I_{\Sigma}(t)$ for which the maximum of function $C$ was reached. The moment of the main shock of the Wenchuan Earthquake is marked by the blue shaded triangles. (e) Demonstrates that no noticeable TEC response to that earthquake was found in far-field regions. The TEC series $\mathrm{d} I(t)$ for GPS satellite PRN22 and GPS sites TCMS (KGN) and 1152 (GEONET) are shown in black and blue, respectively. The accumulated series $\mathrm{d} I_{\Sigma}(t)$ obtained as a result of array processing for KGN and GEONET networks is shown in $(e)$ by a red line.

a result of summation of TEC series for each separate LOS of the array with the shifts maximizing the value of energy of the accumulated signal. At a modelling stage the TEC series shifts are calculated within the model of the plane or spherical wavefront of the perturbation propagating with constant velocity: 


$$
\mathrm{d} I_{\Sigma}(t)=\mathrm{d} I_{0}(t)+\sum_{i=1}^{M} \mathrm{~d} I_{i}\left(t-\tau_{i}\right)
$$

where $i=1,2, \ldots, M$ are the TEC series numbers, $\mathrm{d} I_{0}(t)$ is a central series of arrays to which all other series are summed, $\tau_{i}$ is the time delay shift of the $i$ th series; $M$ is the number of elements in the GPS-array. The processing for the spherical wavefront is described in detail by Kiryushkin and Afraimovich (2007). For the spherical wavefront approximation the QOA algorithm allows us to determine the altitude $H_{e}$ of a secondary source of the wave perturbation registered by GPS-array.

For the spherical wavefront approximation, we used SIPs for all 85 GPS sites. The accumulated series $\mathrm{d} I_{\Sigma}(t)$ for the experiment (black line) and the model (red line) are presented in figure $3(f)$. We can see insufficient correspondence between the experiment and model. It agrees with a very low maximal value of $C(V)_{\max }$ of about 0.25 although $C(V)_{\max }$ corresponds to the value of $V$ obtained by plane wavefront approximation.

The distribution of maximum amplitude $\mathrm{d} I_{\max }$ on corresponding direction azimuth $\alpha$ from the epicentre to all 85 SIPs is shown in figure 4(b). A grey line marks the direction diagram $\mathrm{d} I_{\max }(\alpha)$. We found that the maximal response to the main shock was registered in the southeast sector. It determines the choice of GPS sites for the plane wavefront approximation. The location of the SIPs at the time $t_{\max }$ for all sites used for the plane wavefront model is marked in figure 2 by dots (in all 40 SIPs). The corresponding distribution of amplitude $\mathrm{d} I_{\max }$ on distance $D$ from the epicentre along the great circle arc is shown in figure 4(a). One can see that the amplitude decreases with distance $D$ very quickly - five times at a distance of 700-1000 km.

For the plane wavefront, we form the GPS-array direction diagram and direct it in the phase space $[\alpha, V]$, when assigning values of the arrival direction $\alpha$ and the propagation velocity $V$ and carrying out the coherent accumulation of TEC series $\mathrm{d} I(t)$. The accumulated series $\mathrm{d} I_{\Sigma}(t)$ for the experiment (black line) and the plane waveform model (red line) are presented in figure $3(c)$. We can see full correspondence between the experiment and the model. The 2-dimensional criterion function $C(\alpha, V)$ for the plane wavefront model is shown in figure 5; the thin horizontal line marks the cross section of $C(\alpha, V)$ corresponding to the dependence $C(V)$ for the plane waveform shown in figure 4(c). A maximum value $C(\alpha, V)_{\max }$ of about 0.8 (marked by a red cross) corresponds to the values of velocity $V=560 \mathrm{~m} \mathrm{~s}^{-1}$ and azimuth $\alpha=160^{\circ}$ of wavefront propagation. It should be noted that these values coincide exactly with the ones obtained using the D1 method.

We speculated that the ionospheric disturbance may have been registered over the Korean peninsula and the Japanese islands during the range 07:00-09:00 UT (proceeding from the perturbation velocity value calculated in the near zone of the earthquake epicentre). Unfortunately, no noticeable TEC response to that earthquake was found in far-field regions of South Korea and Japan at this time. The filtered vertical TEC series $\mathrm{d} I(t)$, obtained for GPS satellite numbers PRN22 and GPS sites TCMS $(\mathrm{KGN})$ and 1152 (GEONET) are shown in figure 3(e) in black and blue, respectively.

We used the QOA algorithm for GPS-TEC data processing for KGN and GEONET networks and tried to detect the co-seismic ionospheric perturbations and to determine their spatio-temporal parameters. However, the experimental stage has shown that in the phase summation of the TEC series, during array processing the coherent fluctuations from the same source in TEC series were not found (accumulated series $\mathrm{d} I_{\Sigma}(t)$ is shown in figure 3(e) with a red line). 


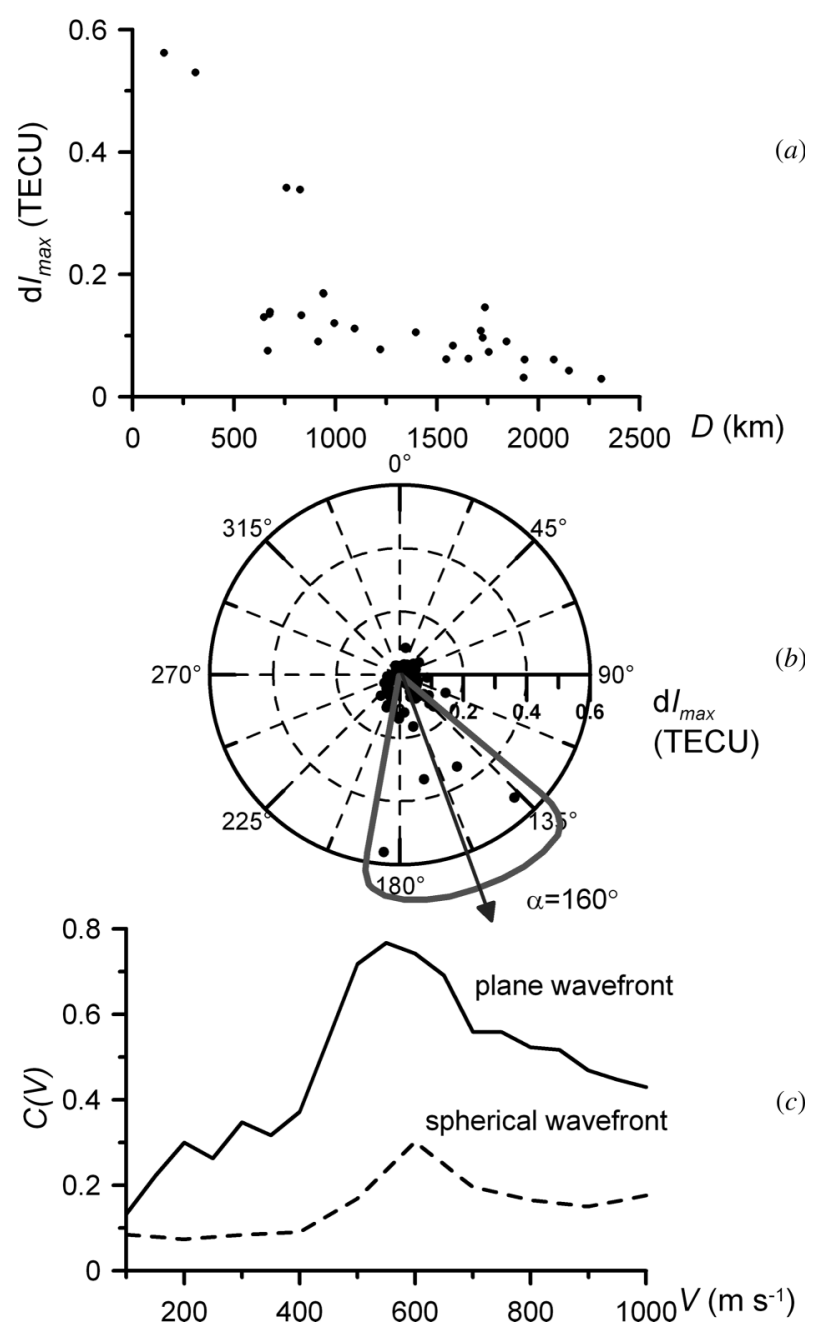

Figure 4. Spatio-temporal parameters of the co-seismic ionospheric perturbation. (a) Distribution of the TEC response amplitude $\mathrm{d} I_{\max }$ to distance $D$ from the epicentre along the great circle arc (40 SIPs). (b) Distribution of maximum amplitude $\mathrm{d} I_{\max }(\alpha)$ on corresponding direction azimuth $\alpha$ from the epicentre to SIPs; maximal response to the main shock was registered in the southeast sector ( 85 SIPs). The grey line marks the direction diagram $\mathrm{d} I_{\max }(\alpha)$. (c) Criterion function $C(V)$ for the plane (solid line) and spherical (dotted line) wavefront approximations.

\section{Comparison with TEC response to other strong earthquakes}

It is very interesting to compare obtained characteristics of the TEC response to the Wenchuan Earthquake with similar parameters for other strong earthquakes. General information about these earthquakes is presented in table 1 (including the time of the main shock $t_{0}$ in universal time (UT), the position of the earthquake epicentre, depth, magnitude, as well as the level of geomagnetic disturbance from the 


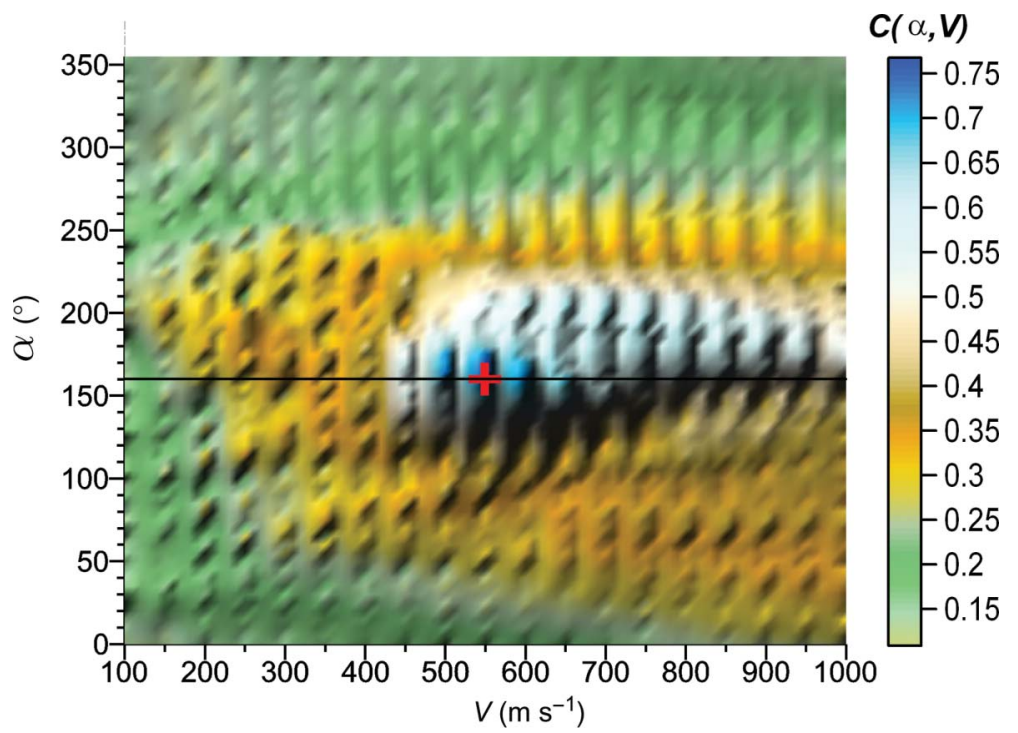

Figure 5. Two-dimensional criterion function $C(\alpha, V)$ for the plane wavefront model; thin horizontal line marks the cross section of $C(\alpha, V)$ corresponding to the dependence $C(V)$ to the plane wavefront shown in figure $4(c)$. Maximum value $C(\alpha, V)_{\max }$ of about 0.8 (marked by a red cross) corresponds to the values of velocity $V=560 \mathrm{~m} \mathrm{~s}^{-1}$ and azimuth $\alpha=160^{\circ}$ of the wavefront propagation.

data on disturbance storm time index (Dst)-variations). It was found that the deviation of Dst for the selected days was quite moderate, which enabled the SAWs to be identified.

General information about TEC response parameters is presented in table 1, including the $T$-SAW period; $A$ - the maximum amplitude of absolute $\mathrm{d} I$ (TECU), and relative $\mathrm{d} I / I(\%)$, TEC deviation for selected LOS; the type of wavefront (plane or spherical); the horizontal component of the phase velocity $V$; the azimuth $\alpha$ of the SAW vector; the altitude $H_{e}$ of a secondary source of the wave perturbation registered by GPS-array, for the spherical wavefront approximation. The second column also shows the references. The relative amplitude $\mathrm{d} I / I_{0}$ is determined by normalization of the $\mathrm{d} I$ to the background $I_{0}$ value, where $I_{0}$ is the absolute vertical TEC obtained with a 2-hour time resolution from the global TEC maps in the Ionospheric Map Exchange ASCII (IONEX) format, so called Global Ionospheric Maps (GIM, ftp://cddisa.gsfc.nasa.gov/ pub/gps/products/ionex/).

The absolute $\mathrm{d} I$ and relative amplitude $\mathrm{d} I / I_{0}$ of TEC deviation increase with the increasing earthquake magnitude. One can see from table 1 that the estimations of perturbation propagation velocity $V$ and altitude $H_{e}$ of a source of the perturbation, whatever the type of source of seismo-ionospheric disturbance, are in agreement with a theory according to which coseismic atmospheric disturbance propagates within a narrow cone of zenith angles up to ionospheric heights and then diverges in the form of a spherical wave with the radial velocity close to the speed of sound at these heights (Afraimovich et al. 2006, Kiryushkin and Afraimovich 2007).

Here, we want to compare TEC response observed during the Wenchuan Earthquake with the Great Sumatra Earthquake that occurred on 26 December 2004. The ionospheric disturbance registered after the earthquake was very powerful (Heki 


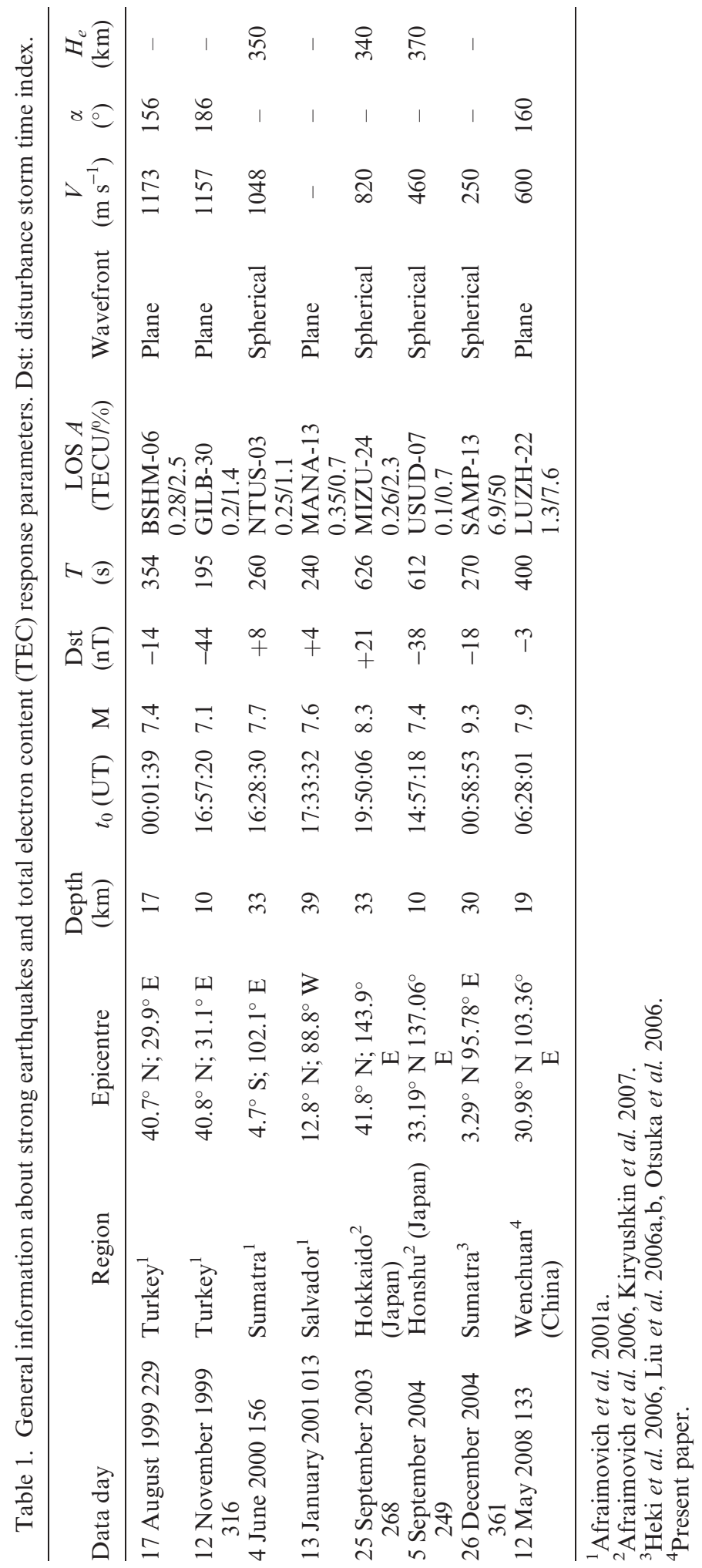


et al. 2006, Liu et al. 2006a,b, Otsuka et al. 2006) and is commensurable with the response to the Wenchuan Earthquake. Otsuka et al. (2006) found anisotropy in the TEC variations with respect to the azimuth from the epicentre. Large TEC enhancements (1 TECU) were observed north of the epicentre. South of the epicentre, on the contrary, TEC was only slightly enhanced, by 0.6 TECU, and TEC enhancement was not seen east of the epicentre. However, in this case the authors connected the observed anisotropy of the TEC variations with anisotropy in the response of the electron density variation to the neutral motion of acoustic waves in oblique geomagnetic field lines.

Afraimovich et al. (2001d) compared the TEC disturbances causing the shock-acoustic waves generated during rocket launchings (Afraimovich et al. 2001c), earthquakes and explosions, using our GPS-array method (Afraimovich et al. 2001a) for determining SAW parameters (including angular characteristics of the wave vector, and the SAW phase velocity). They analysed the launches of Proton, Soyuz, Dnepr, Zenit-2, Space Shuttle and Shenzhou-2 rockets from the Baikonur (Kazakhstan), Kennedy Space Center (USA) and Jiuquan (China) cosmodromes in 1998-2001, as well as the earthquakes in Turkey on 17 August and 12 November 1999, in Southern Sumatra (4 June 2000), and off the coast of Central America (13 January 2001), and during the New Mexico power explosion on 6 June 1993. It was shown that, whatever the type of source, the impulsive TEC disturbance has the character of a classical $\mathrm{N}$-wave with a period of 180-360 s, and with its amplitude exceeding the level of background fluctuations under moderate geomagnetic conditions by a factor of $2-5$ as a minimum. The phase velocity $\left(600-1200 \mathrm{~m} \mathrm{~s}^{-1}\right)$ approaches the sound velocity at the height of the ionospheric F-region maximum. So, the parameters of ionospheric response on impulsive impact are determined not only by source characteristics but substantially by the properties of the ionospheric channel of the shock-acoustic wave propagation.

\section{Conclusions}

We registered near-field GPS TEC response to the Wenchuan Earthquake on 12 May 2008. We found that intensive $N$-shaped shock-acoustic waves with a plane waveform and with a half-period of about 200 s propagated south-eastwards with a velocity of $580 \mathrm{~m} \mathrm{~s}^{-1}$ for a distance of about $1000 \mathrm{~km}$ from the epicentre. The wavefront of the $N$ shaped disturbances was parallel with the earthquake rupture direction (from southwest to northeast). The main directional lobe of shock-acoustic wave emitter is directed southeastwards, i.e. transversely to the rupture. We believe that the above properties of TEC response are determined by the geodynamics of the Wenchuan Earthquake. No noticeable TEC response to that earthquake was found in far-field regions of South Korea and Japan. Detailed investigation of that phenomenon and corresponding modelling are the aims of our future works.

We compared TEC response to the 2008 Wenchuan Earthquake with other strong earthquakes. Comparison shows that whatever the type of source of seismoionospheric disturbances, the results obtained are in agreement with a theory according to which co-seismic atmospheric disturbance propagates within a narrow cone of zenith angles up to ionospheric heights and then diverges in the form of a spherical wave with the radial velocity close to the speed of sound at these heights.

\section{Acknowledgments}

The authors are grateful to S.V. Voeykov for help with primary data processing. The authors wish to thank members of the Crustal Movement Observation Network of 
China, the Geonet and the KGN networks for GPS data used in this paper. The work was supported by the interdisciplinary integral project of SB RAS N 56 'Seismoionospheric and seismoelectromagnetic processes in Baikal Rift Zone', the RFBR-GFEN grant no. 06-05-39026 and RFBR grant no. 07-05-00127; by the Japanese Society for the Promotion of Science (JSPS); by the National Natural Science Foundation of China (grant no. 40774090 and grant no. 40636032); by the National Important Basic Research Project (2006CB806306) and the Korea Meteorological Administration Research and Development Program under grant no. CATER 2006-3104. The authors are grateful to Ramesh Singh for his comments and suggestions for improving the earlier version of the manuscript.

\section{References}

Afraimovich, E.L., 2000, GPS global detection of the ionospheric response to solar flares. Radio Science, 35, pp. 1417-1424.

Afraimovich, E.L., Perevalova, N.P., Plotnikov, A.V. and Uralov, A.M., 2001a, The shockacoustic waves generated by the earthquakes. Annales Geophysicae, 19, pp. 395-409.

Afraimovich, E.L., Kosogorov, E.A., Lesyuta, O.S., Ushakov, I.I. and Yakovets, A.F., 2001b, Geomagnetic control of the spectrum of traveling ionospheric disturbances based on data from a global GPS network. Annales Geophysicae, 19, pp. 723-731.

Afraimovich, E.L., Kosogorov, E.A., Perevalova, N.P. and Plotnikov, A.V., 2001c, The use of GPS-arrays in detecting shock-acoustic waves generated during rocket launchings. Journal of Atmospheric and Solar-Terrestrial Physics, 63, pp. 1941-1957.

Afraimovich, E.L., Kosogorov, E.A., Perevalova, N.P. and Plotnikov, A.V., 2001d, Comparison of TEC response of the shock-acoustic waves generated during rocket launchings, by earthquakes and explosions. Proceedings of International Beacon Satellite Symposium, 4-6 June 2001, Boston College, Institute for Scientific Research, Chestnut Hill, MA, USA, pp. 373-377 (Boston, MA: Boston College Press).

Afraimovich, E.L., Kiryushin, V.V. and Perevalova, N.P., 2002a, Determination of the characteristics of ionospheric perturbations in the near-field region of an earthquake epicenter. Journal of Communications Technology and Electronics, 47, pp. 739-747.

Afraimovich, E.L., Kosogorov, E.A. and Plotnikov, A.V., 2002b, Shock-acoustic waves generated during rocket launchings and earthquakes. Cosmic Research, 40, pp. 261-275.

Afraimovich, E.L., Astafyeva, E.I. and Kiryushinin, V.V., 2006, Localization of the source of ionospheric disturbance generated during an earthquake. International Journal of Geomagnetism and Aeronomy, 6, GI2002, doi: 10.1029/2004GI000092.

CAlais, E. and Minster, J.B., 1995, GPS detection of ionospheric perturbations following the January 1994, Northridge earthquake. Geophysical Research Letters, 22, pp. 1045-1048.

Calais, E. and Minster, J.B., 1998, GPS, earthquakes, the ionosphere, and the Space Shuttle. Physics of the Earth and Planetary Interiors, 105, pp. 167-181.

Heki, K., Otsuka, Y., Choosakul, N., Hemmakorn, N., Komolmis, T. and Maruyama, T., 2006, Detection of ruptures of Andaman fault segments in the 2004 great Sumatra earthquake with coseismic ionospheric disturbances. Journal of Geophysical Research, 111, B09313, doi: 10.1029/2005JB004202.

Ji, C. and Hayes, G., 2008, Finite fault model of the May 12, 2008, Mw 7.9 Eastern Sichuan, China Earthquake. Report, United States Geological Survey, Reston, VA. Available online at http://earthquakeusgs.gov/earthquakes/eqinthenews/2008/us2008ryan/finite_fault.php.

JIN, S.G., and PARK, J.U., 2007, GPS ionospheric tomography: a comparison with the IRI-2001 model over South Korea. Earth Planets and Space, 59, pp. 287-292.

Kiryushinin, V.V. and Afraimovich, E.L., 2007, Determining the parameters of ionospheric perturbation caused by earthquakes with using the quasi-optimum algorithm of spatiotemporal processing of TEC measurements. Earth, Planets and Space, 59, pp. 267-278. 
KLOBuchar, J.A., 1986, Ionospheric time-delay algorithm for single-frequency GPS users. IEEE Transactions on Aerospace and Electronics System, 23, pp. 325-331.

LIN, A., REN, Z., JiA, D. and Wu, X., 2009, Co-seismic thrusting rupture and slip distribution produced by the $2008 \mathrm{Mw} 7.9$ Wenchuan earthquake, China. Tectonophysics, doi: 10.1016/j.tecto.2009.02.014.

Liu, J.Y., Tsai, Y.B., Chen, S.W., Lee, C.P., Chen, Y.C., Yen, H.Y., Chang, W.Y. and Liu, C., 2006a, Giant ionospheric disturbances excited by the M9.3 Sumatra earthquake of 26 December 2004. Geophysical Research Letters, 33, L02103, doi: 10.1029/ 2005 GL023963.

Liu, J.Y., Tsai, Y.B., Ma, K.F., Chen, Y.I., Tsai, H.F., Lin, C.H., Kamogawa, M. and Lee, C.P., 2006b, Ionospheric GPS total electron content (TEC) disturbances triggered by the 26 December 2004 Indian Ocean tsunami. Journal of Geophysical Research, 111, A05303, doi: 10.1029/2005JA011200.

Otsuka, Y., Kotake, N., Tsugawa, T., Shiokawa, K., Ogawa, T., Effendy, Saito, S., Kawamura, M., Maruyama, T., Hemmakorn, N. and Komolmis, T., 2006, GPS detection of total electron content variations over Indonesia and Thailand following the 26 December 2004 earthquake. Earth Planets and Space, 58, pp. 159-165.

Yu, T., MaO, T., WAng, Y. and WAng, J., 2009, Study of the ionospheric anomaly before the Wenchuan earthquake. Chinese Science Bulletin, 54, pp. 1-7.

Zhao, B., Wang, M., Yu, T., Wan, W., Lei, J., Liu, L. and Ning, B., 2008, Is an unusual large enhancement of ionospheric electron density linked with the 2008 great Wenchuan earthquake? Journal of Geophysical Research, 113, A11304, doi: 10.1029/ 2008JA013613. 